\title{
Cultivo de Batis maritima, Sarcocornia ambigua e Sporobolus virginicus com água residuária do cultivo de Litopenaeus vannamei
}

\author{
Roberta Moriconi Freire Schardong 1* \\ Oriel Herrera Bonilla ${ }^{2}$ \\ Sandra Tédde Santaella ${ }^{1}$ \\ ${ }^{1}$ Universidade Federal do Ceará \\ Avenida da Abolição, 3207, Meireles, CEP 60165-081, Fortaleza - CE, Brasil \\ ${ }^{2}$ Universidade Estadual do Ceará, Fortaleza - CE, Brasil \\ * Autor para correspondência \\ mfs.roberta@gmail.com
}

Submetido em 01/11/2017

Aceito para publicação em 26/03/2018

\section{Resumo}

As águas residuárias de carcinicultura descartadas diretamente no ambiente podem provocar diversos impactos para a estabilidade dos ecossistemas adjacentes. Nesta perspectiva, este trabalho compara: o crescimento de plantas halófitas cultivadas com água residuária do cultivo de camarão e com água bruta de um estuário; a interação entre o cultivo integrado de plantas halófitas com camarões em sistemas de recirculação de água; e as medidas de variáveis físico-químicas das águas residuárias antes e após serem submetidas a um sistema de tratamento com plantas halófitas. Para tanto, foram realizados dois experimentos de bancada utilizando caixas de fibra para o cultivo das halófitas. Em ambos os experimentos foram realizadas medições semanais do crescimento e das massas inicial e final das plantas. As taxas de crescimento relativo e absoluto foram mensuradas para a massa e a altura das espécies vegetais. As taxas de crescimento absoluto para a massa da espécie Batis maritima dos sistemas um e dois do experimento 1 e do sistema com plantas do experimento 2 foi, respectivamente, $1,12 \mathrm{~cm} / \mathrm{d}, 4,40 \mathrm{~cm} / \mathrm{d}$ e $3,55 \mathrm{~cm} / \mathrm{d}$. Os dados indicaram que halófitas cultivadas com águas residuárias de carcinicultura apresentam crescimento maior, assim como são reduzidos os nutrientes do meio de cultivo na presença das plantas.

Palavras-chave: Águas residuárias; Plantas halófitas; Recirculação de água

\section{Abstract}

Cultivating Batis maritima, Sarcocornia ambigua and Sporobolus virginicus with wastewater from the cultivation of Litopenaeus vannamei. Shrimp wastewater discarded directly into the environment can impact the stability of adjacent ecosystems. This study evaluated the following: the growth of halophytes cultivated with shrimp culture water and water from an estuary; the interaction between the integrated cultivation of halophytes and shrimp in water recirculation systems; and physicochemical variables of wastewater before and after being treated with halophytes. Two bench experiments were carried out using fiber boxes to cultivate the halophytes. In both experiments, growth and initial and final mass of the plants were measured weekly. The 
relative and absolute growth rates were measured for the mass and height of the plant species. The absolute growth rates for Batis maritima of systems one and two of the first experiment, and of the system of plants of the second experiment, were $1.12 \mathrm{~cm} / \mathrm{d}, 4.40 \mathrm{~cm} / \mathrm{d}$ and $3.55 \mathrm{~cm} / \mathrm{d}$, respectively. The data indicated that halophytes cultivated with shrimp wastewater had higher growth and that the nutrients of the culture medium were reduced in the presence of plants.

Key words: Halophytic plants; Recirculation; Wastewater

\section{Introdução}

Halófitas são plantas promissoras para diferentes tipos de aplicações devido à composição química e características fisiológicas especiais que possibilitam o crescimento em ambientes salinos (BUHMANN; PAPENBROCK, 2013). Têm capacidade para acumular metais pesados, tornando-as propícias para fitoextração e fitoestabilização em solos contaminados (VAN OOSTEN; MAGGIO, 2015).

Pesquisas indicam o uso de halófitas para complementação da dieta de caprinos (SHAER, 2010); produção de subprodutos de valor nutricional, medicinal e artesanal para o ser humano (KSOURI et al., 2012); redução da salinidade do solo (QADIR et al., 2007; HASANUZZAMAN et al., 2014); tratamento de águas residuárias, possibilitando o reúso; dar origem a subprodutos na indústria química (LIETH et al., 2008); produção de biodiesel (SOTIROUDIS et al., 2010); e captura de $\mathrm{CO}_{2}$ da atmosfera (LIETH et al., 2008).

A importância descrita na literatura para a espécie Batis maritima (Linnaeus) inclui: a proteção de zonas costeiras e estabilização de substrato; a cultura potencial para solos salgados e salinos; o valor nutritivo, por conter $17,3 \%$ de proteína bruta e $25 \%$ de óleo; o uso de suas folhas em saladas, em Porto Rico, e na medicina popular para tratamento de gota, eczema, psoríase, reumatismo, sangue e distúrbios da tireoide; o fornecimento de nutrição e cobertura para animais selvagens; o uso para curar infecções cutâneas, na Península de Yucatán no México (LONARD et al., 2011); e o fato de suas sementes serem ricas em aminoácidos, como lisina e metionina (MARCONE, 2003).

A espécie Sporobolus virginicus (C. Linnaeus) K. Kunth, da família Poaceae (Gramineae), é um capim de crescimento estimulado pela presença de sal e tem ampla distribuição nas costas dos hemisférios norte e sul do globo terrestre (LONARD et al., 2013). A espécie é utilizada para controle de erosão em praias e para estabilizar substratos costeiros arenosos; fornece forragem de boa qualidade para gado, cabras, ovelhas e camelos; disponibiliza cobertura e comida para grande diversidade de seres selvagens; é fonte de nutrição para espécies de camarão e caranguejos; no Havaí é usada na medicina popular para tratar aftas, doença da infância e enfraquecimento físico (LONARD et al., 2013).

Sarcocornia ambigua (Michx.) M.A. Alonso \& M.B. Crespo é uma espécie perene da família Chenopodiaceae, que apresenta um grande potencial biotecnológico como uma cultivar que pode ser irrigada com água salgada (D’OCA et al., 2012). Esse gênero encontra-se distribuído na América do Sul, ocorrendo da costa da Venezuela até a foz do rio Plata, na Argentina (D'OCA et al., 2012). Estudos indicaram potencial dessa espécie como fonte valiosa de antioxidantes naturais e nutrientes para uso nas indústrias alimentar e farmacêutica (BERTIN et al., 2014).

De forma geral, as plantas halófitas apresentam diversidade estrutural de constituintes químicos (vitaminas, minerais e compostos bioativos) pela complexidade fisiológica. São plantas que se adaptaram a ecossistemas costeiros e, portanto, seu ciclo de vida ocorre em ambientes com elevada concentração salina, entre 100 a 500 mmol.L ${ }^{-1}$ de $\mathrm{NaCl}$ (FLOWERS; COLMER, 2008).

As características fisiológicas específicas de plantas halófitas possibilitam a associação do cultivo dessas espécies com atividades de carcinicultura, tanto para produção de biomassa vegetal quanto para tratamento da água residuária, de acordo com estudos que vêm sendo realizados com sucesso (PINHEIRO et al., 2017). 
A carcinicultura, embora gere empregos, impulsiona conflitos relacionados à poluição hídrica, à salinização dos lençóis freáticos, à destruição de manguezais, aos riscos de introdução de espécies exóticas e de difusão de epidemias e à desestruturação das comunidades de pescadores artesanais; além disso, o lançamento de dejetos oriundos do sistema produtivo tem excedido a capacidade de assimilação dos ecossistemas nos quais os empreendimentos estão inseridos (FABIANO, 2004).

As águas residuárias de carcinicultura descartadas diretamente no ambiente, sem tratamento prévio, contêm restos de alimentos, fezes e fragmentos de animais, podendo causar a deterioração das águas dos corpos receptores ou eutrofização (RIBEIRO et al., 2014). Consequentemente, podem ocasionar modificação do habitat, resultando em perda de flora e fauna (RIBEIRO et al., 2014), sendo necessário buscar alternativas de recirculação e destino adequado para essas águas residuárias.

Usualmente, o tratamento da água residuária para remoção dos poluentes é feito em tanques ou bacias de sedimentação e wetlands construídas (BIUDES, 2007). Wetlands construídas são sistemas desenhados para utilizar processos naturais para remoção de poluentes da água residuária (KIVAISI, 2001), atuando, principalmente, na remoção de nitrogênio e fósforo da água residuária por meio da sedimentação, precipitação química e adsorção (BRASKERUD, 2002). As wetlands que utilizam plantas halófitas possibilitam o tratamento de águas residuárias salinizadas combinado à produção de culturas secundárias valiosas (BUHMANN; PAPENBROCK, 2013).

Estudo que avaliou o cultivo integrado de $S$. ambigua e o camarão L. vannamei, em sistema aquapônico, concluiu que o aproveitamento do nitrogênio fornecido ao sistema foi mais eficiente no tratamento com plantas; assim como a qualidade da água na presença das plantas manteve-se adequada para o cultivo dos camarões (PINHEIRO et al., 2017).

Dessa forma, este trabalho visou comparar o crescimento das plantas halófitas $B$. maritima e Sporobolus virginicus cultivadas com água residuária do cultivo de camarão L. vannamei e com água bruta do estuário do rio Pacoti (CE); verificar a interação entre o cultivo das halófitas B. maritima, Sarcocornia ambigua e $S$. virginicus com camarões em sistemas de recirculação de água; e determinar a qualidade físico-química das águas residuárias antes e após serem submetidas ao sistema contendo as halófitas.

\section{Material e Métodos}

\section{Funcionamento dos experimentos}

O estudo foi realizado no Centro de Estudos em Aquicultura Costeira/CEAC, Instituto de Ciências do Mar da Universidade Federal do Ceará, contemplando dois experimentos, o primeiro teve cento e onze dias de duração, em 2016, e o segundo, setenta dias de duração, em 2017.

No experimento 1 foram montados três sistemas de material de fibra $(0,90 \mathrm{~m}$ de comprimento $\mathrm{x} 0,48 \mathrm{~m}$ de largura $\mathrm{x}$ 0,11 $\mathrm{m}$ de profundidade), contendo cinco compartimentos, ligados por mangueiras de silicone a depósitos de plásticos localizados em cada uma das extremidades (Figura 1). O primeiro sistema recebia água filtrada do rio Pacoti e os outros dois, águas residuárias da criação de camarão. Os sistemas foram protegidos, com lona cinza, da chuva e da radiação solar direta.

FIGURA 1: Esquema do primeiro experimento.

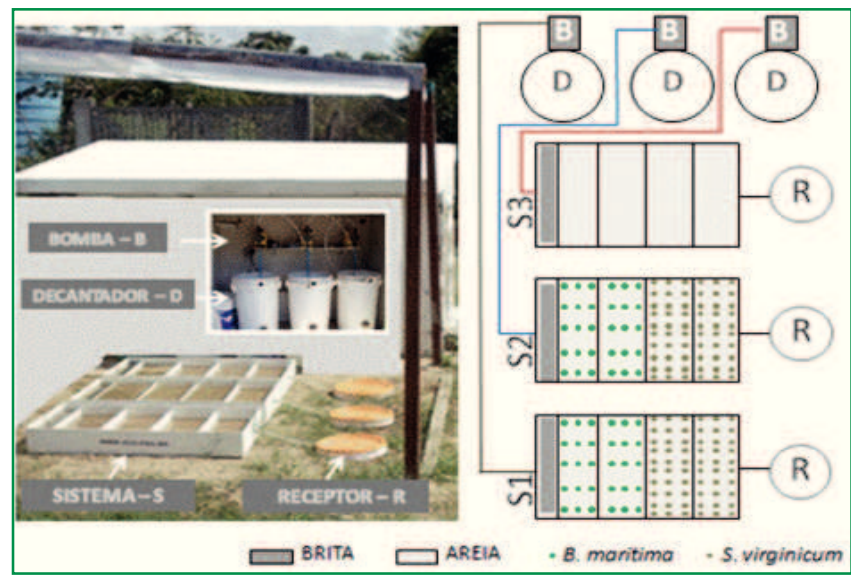

Quarenta e quatro litros de água residuária/ água bruta eram colocados nos decantadores (D), permanecendo por $1 \mathrm{~h}$. Depois as bombas eram ligadas com ciclos de aplicação semanais, sendo o tempo de 
aplicação de dois a três dias até a passagem de $35 \mathrm{~L}$ com vazão de $12 \mathrm{~mL} / \mathrm{min}$ e o tempo de descanso de quatro a cinco dias. Na parte posterior, o recipiente receptor $(\mathrm{R})$ foi enterrado e mantido tampado recebendo por gravidade a água residu ria dos sistemas. $\mathrm{O}$ volume acumulado nos receptores era medido e, posteriormente, descartado antes do início do ciclo de aplicação seguinte.

Durante as oito primeiras semanas as guas do estu rio e residu ria foram diluídas com gua pot vel da rede de abastecimento pública nas proporções de gua pot vel: gua do estu rio ou residu ria de $25 \%: 75 \%$, nas três primeiras semanas, $50 \%: 50 \%$, nas quatro semanas seguintes, e $25 \%: 75 \%$, na oitava semana. As plantas foram colhidas em locais de cultivo a céu aberto, expostas ao regime de chuvas e descartes eventuais das guas residu rias. Posteriormente, as mudas de estaca foram preparadas com gua doce, sendo necess ria a diluição da gua salgada para aclimatação das guas.

O primeiro compartimento dos sistemas foi preenchido com brita $\mathrm{n}-0$ de granulometria $4,8 \mathrm{~mm}$ a 9,5 mm, para retenção de partículas. O substrato dos compartimentos seguintes foi areia média comercial de granulometria entre $0,42 \mathrm{~mm}$ e $2,00 \mathrm{~mm}$, sendo que nos sistemas um e dois foram plantadas trinta mudas de $B$. maritima nos dois primeiros compartimentos e sessenta mudas de $S$. virginicus nos dois últimos. O terceiro sistema não recebeu plantas.

O experimento 2 foi com recirculação de água residu ria de tanques (recipientes de pl stico de 61 L) com 15 camarões (Figura 2). A água dos tanques era aplicada aos sistemas com vazão de $12 \mathrm{~mL} / \mathrm{min}$, retornando aos tanques por gravidade. O primeiro sistema estava vazio, passando livremente a gua residu ria. Os sistemas dois e três foram preenchidos com brita $\mathrm{n}^{\mathrm{0}} 0$ no primeiro compartimento e areia média comercial nos outros compartimentos. No terceiro sistema foram cultivadas em fileiras alternadas trinta $\mathrm{e}$ seis mudas de $B$. maritima, doze mudas de $S$. ambigua e doze mudas de $S$. virginicus.

\section{Determinação das variáveis físico- químicas das águas}

Os parâmetros: temperatura, sólidos totais dissolvidos, salinidade, $\mathrm{pH}$ e oxigênio dissolvido foram medidos com o aparelho HI98194 Multiparâmetro Portátil de $\mathrm{pH} / \mathrm{EC} / \mathrm{DO}$. No experimento 1, as medidas foram realizadas na entrada e saída de cada sistema, uma vez por semana. No experimento 2, as medidas foram feitas nos tanques, diariamente.

Ortofosfato e sólidos suspensos foram determinados, uma vez por semana, para o experimento 1

FIGURA 2: Esquema do segundo experimento.

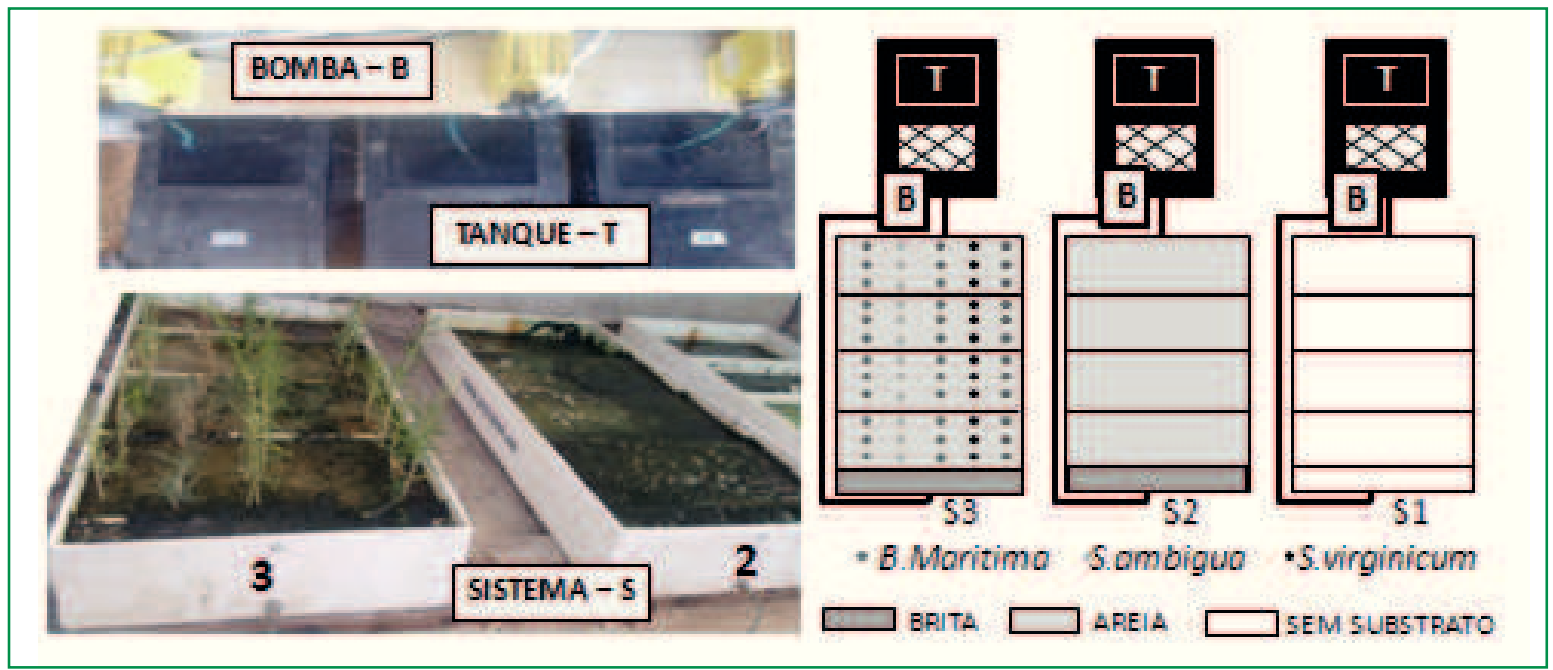


e quinzenalmente para o experimento 2, sendo, no experimento 1, coletadas amostras na entrada e na saída de cada sistema. Após a coleta, a determinação de ortofosfato foi com testes Hach-Lange comerciais, utilizando um espectrofotômetro (DR 2800 Spectrophotometer, Hach Company, Loveland, EUA). Os sólidos suspensos também foram medidos com o uso do espetrofotômetro.

Para o experimento 2, as coletas das amostras foram realizadas nos tanques, sendo determinados, além do ortofosfato e sólidos suspensos, a amônia, o nitrito e o nitrato com testes Hach-Lange comerciais, utilizando um espectrofotômetro (DR 2800 Spectrophotometer, Hach Company, Loveland, EUA).

\section{Halófitas}

Estacas de B. maritima, $S$. ambigua e $S$. virginicus foram coletadas no CEAC e enraizadas em bandejas, com substrato comercial para mudas, durante quatro semanas. Antes de serem plantadas nos sistemas, as mudas foram pesadas em balança digital com duas casas decimais e suas alturas foram medidas com régua de alumínio, considerando a distância do colo da planta até o ponto de inserção da última folha. Além disso, as Taxas de Crescimento Relativo (TCR) e Absoluto (TCA) foram calculadas para a altura e a massa. As taxas para massa foram calculadas com base na biomassa úmida.

No experimento 1, as plantas foram transplantadas para os sistemas na quarta semana do início do experimento. No experimento 2, o transplante das mudas foi no início do experimento.

Semanalmente, a altura de cada planta era medida e registrada. Após o término dos experimentos, as plantas foram pesadas úmidas e secas. A secagem foi realizada em estufa à temperatura de $56^{\circ} \mathrm{C}$ durante quatro dias.

\section{Litopenaeus vannamei}

O povoamento dos tanques do experimento 2 foi com L. vannamei, que foram pesados no início e ao final do experimento. A ração era ofertada duas vezes ao dia, às $08 \mathrm{~h} 00 \mathrm{e}$ às $16 \mathrm{~h} 00$. A sobra da ração era contada e pesada. A ração continha $35 \%$ de proteína bruta na base natural ( $10 \%$ de umidade).

\section{Tratamento de dados}

A comparação entre os dados das variáveis determinadas na entrada e na saída de cada sistema do experimento 1 foi feita com teste $T$ para amostras pareadas, quando as amostras eram normais e homogêneas, e com teste de Wilcox para amostras com distribuição anormal ou não homogêneas. $\mathrm{O}$ teste de normalidade adotado foi de Shapiro-Wilk, e o Teste F foi usado para medir a homogeneidade entre as amostras.

Os mesmos testes de hipóteses foram adotados para comparar os dados dos valores dos parâmetros na saída dos sistemas dois e três do experimento 1, como amostras independentes, e para os parâmetros medidos em cada tanque do experimento 2 .

O teste de Wilcox foi usado para verificar se havia diferença significativa no crescimento da altura entre as plantas irrigadas com gua e aquelas irrigadas com água residuária do experimento 1 , depois de testados os pressupostos para normalidade e homogeneidade. Todos os testes de hipótese foram analisados no R-Studio (RStudio, versão 3.2.4, Boston, USA). O valor máximo $p$ aceitável para verificação de significância foi de 0,05 .

\section{Resultados}

\section{Variáveis físico-químicas}

Os valores das vari veis salinidade, sólidos totais suspensos e ortofosfato, entre a entrada e a saída de cada sistema no experimento 1 , apresentaram diferenças significativas. Para sólidos suspensos a diferença significativa ocorreu apenas no sistema um (Tabela 1). Salinidade e sólidos suspensos totais foram maiores na saída dos sistemas, enquanto para sólidos suspensos e ortofosfato os valores foram maiores na entrada dos sistemas dois e três. 
TABELA 1: Comparação das variáveis físico-químicas da água residuária do L. vannamei para amostras pareadas.

\begin{tabular}{|c|c|c|c|c|c|c|}
\hline & \multicolumn{2}{|c|}{ Sistemas um } & \multicolumn{2}{|c|}{ Sistemas dois } & \multicolumn{2}{|c|}{ Sistemas três } \\
\hline & Entrada & Saída & Entrada & Saída & Entrada & Saída \\
\hline Temperatura $\left({ }^{\circ} \mathrm{C}\right)$ & $\begin{array}{c}38,2 \pm 4,9 \\
(29,4-44,0)\end{array}$ & $\begin{array}{c}37,0 \pm 4,6(*) \\
(28,9-44,6)\end{array}$ & $\begin{array}{c}36,6 \pm 4,6 \\
(29,2-44,7)\end{array}$ & $\begin{array}{c}36,7 \pm 4,7(*) \\
(28,9-43,8)\end{array}$ & $\begin{array}{c}36,6 \pm 4,6 \\
(29,2-44,7)\end{array}$ & $\begin{array}{c}36,5 \pm 4,6(*) \\
(29,1-44,0)\end{array}$ \\
\hline $\mathrm{pH}$ & $\begin{array}{c}8,1 \pm 0,4 \\
(6,8-8,2)\end{array}$ & $\begin{array}{c}8,0 \pm 0,3(*) \\
(7,3-8,4)\end{array}$ & $\begin{array}{c}8,1 \pm 0,2 \\
(7,8-8,5)\end{array}$ & $\begin{array}{c}8,1 \pm 0,3(*) \\
(7,3-8,6)\end{array}$ & $\begin{array}{c}8,1 \pm 0,2 \\
(7,8-8,5)\end{array}$ & $\begin{array}{c}8,2 \pm 0,3(*) \\
(7,3-8,6)\end{array}$ \\
\hline Salinidade (psu) & $\begin{array}{l}25,7 \pm 9,3 \\
(7,6-36,7)\end{array}$ & $\begin{array}{c}35,9 \pm 11,0(* *) \\
(12,8-62,5)\end{array}$ & $\begin{array}{l}26,3 \pm 11,3 \\
(7,1-41,4)\end{array}$ & $\begin{array}{c}38,0 \pm 14,3(* *) \\
(12,2-67,0)\end{array}$ & $\begin{array}{l}26,3 \pm 11,3 \\
(7,1-41,4)\end{array}$ & $\begin{array}{c}39,5 \pm 13,1(* *) \\
(12,8-64,0)\end{array}$ \\
\hline $\begin{array}{l}\text { Oxigênio } \\
\text { dissolvido }(\mathrm{mg} / \mathrm{L})\end{array}$ & $\begin{array}{c}2,9 \pm 0,8 \\
(1,2-3,9)\end{array}$ & $\begin{array}{c}3,0 \pm 1,2(*) \\
(0,3-1,2)\end{array}$ & $\begin{array}{c}2,8 \pm 0,8 \\
(1,4-4,0)\end{array}$ & $\begin{array}{c}2,7 \pm 1,1(*) \\
(0,2-4,5)\end{array}$ & $\begin{array}{c}2,8 \pm 0,8 \\
(1,4-4,0)\end{array}$ & $\begin{array}{c}3,0 \pm 1,3(*) \\
(0,1-4,9)\end{array}$ \\
\hline $\begin{array}{l}\text { Sólidos suspensos } \\
\text { totais }(\mathrm{mg} / \mathrm{L})\end{array}$ & $\begin{array}{c}20,2 \pm 6,9 \\
(6,7-28,0)\end{array}$ & $\begin{array}{c}27,3 \pm 7,5(* *) \\
(10,7-44,5)\end{array}$ & $\begin{array}{c}21,2 \pm 7,9 \\
(6,4-31,1)\end{array}$ & $\begin{array}{c}28,6 \pm 9,7(* *) \\
(10,2-47,1)\end{array}$ & $\begin{array}{c}21,2 \pm 7,9 \\
(6,4-31,1)\end{array}$ & $\begin{array}{c}29,6 \pm 8,9(* *) \\
(10,7-45,3)\end{array}$ \\
\hline $\begin{array}{l}\text { Sólidos suspensos } \\
(\mathrm{mg} / \mathrm{L})\end{array}$ & $\begin{array}{c}7,6 \pm 8,4 \\
(2,0-33,0)\end{array}$ & $\begin{array}{c}12,1 \pm 12,8(* *) \\
(1,5-45,0)\end{array}$ & $\begin{array}{l}32,0 \pm 15,7 \\
(4,5-66,8)\end{array}$ & $\begin{array}{c}13,0 \pm 17,2(*) \\
(2,0-77,0)\end{array}$ & $\begin{array}{l}32,0 \pm 15,7 \\
(4,5-66,8)\end{array}$ & $\begin{array}{c}16,3 \pm 15,6(*) \\
(1,5-58,5)\end{array}$ \\
\hline $\begin{array}{l}\text { Ortofosfato } \\
(\mathrm{mg} / \mathrm{L})\end{array}$ & $\begin{array}{c}0,2 \pm 0,3 \\
(0,01-0,3)\end{array}$ & $\begin{array}{c}0,4 \pm 0,1(* *) \\
(0,2-0,4)\end{array}$ & $\begin{array}{l}2,5 \pm 1,3 \\
(0,9-5,0)\end{array}$ & $\begin{array}{c}1,3 \pm 0,5(* *) \\
(0,5-2,0)\end{array}$ & $\begin{array}{l}2,5 \pm 1,3 \\
(0,9-5,0)\end{array}$ & $\begin{array}{c}1,3 \pm 0,7(* *) \\
(0,4-2,2)\end{array}$ \\
\hline
\end{tabular}

Dados médios \pm desvio padrão (mínimo e máximo). Teste $t$ para amostas dependentes, com distribuição normal e homogênea. (*) Não apresenta diferença significativa; $(* *)$ Apresenta diferença significativa $(p<0,05)$.

Os valores obtidos de temperatura, $\mathrm{pH}$, ortofosfato e salinidade da saída dos sistemas dois e três, com o cultivo de plantas e sem o cultivo de plantas, respectivamente, podem ser visualizados na Tabela 2 e na Figura 3.
A média da perda de volume de água, durante as dezenove semanas, foi de $9,0 \mathrm{~L}, 11,1 \mathrm{~L}$ e $11,2 \mathrm{~L}$, respectivamente, no primeiro, segundo e terceiro sistema.

TABELA 2: Comparação das vari veis físico-químicas da gua residu ria do L. vannamei para amostras não pareadas dos sistemas dois e três.

\begin{tabular}{l|c|c|c|c}
\hline & $\begin{array}{c}\text { Sistemas dois e três } \\
\text { (entrada) }\end{array}$ & $\begin{array}{c}\text { Sistema dois } \\
\text { (saída) }\end{array}$ & $\begin{array}{c}\text { Sistema três } \\
\text { (saída) }\end{array}$ & $\begin{array}{c}\text { Teste t } \\
\text { (saída) }\end{array}$ \\
\hline \multirow{2}{*}{ Temperatura $\left({ }^{\circ} \mathrm{C}\right)$} & $\begin{array}{c}36,6 \pm 4,6 \\
(29,2-44,7)\end{array}$ & $\begin{array}{c}36,7 \pm 4,7 \\
(28,9-43,8)\end{array}$ & $\begin{array}{c}36,5 \pm 4,6 \\
(29,1-44,0)\end{array}$ & $(*)$ \\
\hline $\mathrm{pH}$ & $\begin{array}{c}8,1 \pm 0,2 \\
(7,8-8,5)\end{array}$ & $\begin{array}{c}8,1 \pm 0,3 \\
(7,3-8,6)\end{array}$ & $\begin{array}{c}8,2 \pm 0,3 \\
(7,3-8,6)\end{array}$ & $(*)$ \\
\hline \multirow{2}{*}{ Salinidade (psu) } & $\begin{array}{c}26,3 \pm 11,3 \\
(7,1-41,4)\end{array}$ & $\begin{array}{c}38,0 \pm 14,3 \\
(12,2-67,0)\end{array}$ & $\begin{array}{c}39,5 \pm 13,1 \\
(12,8-64,0)\end{array}$ & $(*)$ \\
\hline \multirow{2}{*}{ Oxigênio dissolvido (mg/L) } & $\begin{array}{c}2,8 \pm 0,8 \\
(1,4-4,0)\end{array}$ & $\begin{array}{c}2,7 \pm 1,1 \\
(0,2-4,5)\end{array}$ & $\begin{array}{c}3,0 \pm 1,3 \\
(0,1-4,9)\end{array}$ & $(*)$ \\
\hline \multirow{2}{*}{ Sólidos suspensos totais (mg/L } & $\begin{array}{c}21,2 \pm 7,9 \\
(6,4-31,1)\end{array}$ & $\begin{array}{c}28,6 \pm 9,7 \\
(10,2-47,1)\end{array}$ & $\begin{array}{c}29,6 \pm 8,9 \\
(10,7-45,3)\end{array}$ & $(*)$ \\
\hline \multirow{2}{*}{ Sólidos suspensos (mg/L) } & $\begin{array}{c}32,0 \pm 15,7 \\
(4,5-66,8)\end{array}$ & $\begin{array}{c}13,0 \pm 17,2 \\
(2,0-77,0)\end{array}$ & $\begin{array}{c}16,3 \pm 15,6 \\
(1,5-58,5)\end{array}$ & $(*)$ \\
\hline Ortofosfato (mg/L) & $\begin{array}{c}2,5 \pm 1,3 \\
(0,9-5,0)\end{array}$ & $\begin{array}{c}1,3 \pm 0,5 \\
0,5-2,0)\end{array}$ & $\begin{array}{c}1,3 \pm 0,7 \\
(0,4-2,2)\end{array}$ & $(*)$ \\
\hline
\end{tabular}

Dados médios \pm desvio padrão (mínimo e máximo). Teste $t$ para amostas independentes, com distribuição normal e homogênea. $(*)$ : Não apresenta diferença significativa; $(* *)$ Apresenta diferença significativa $(p<0,05)$. 
FIGURA 3: Comparação da média dos valores de pH e de salinidade na saída dos sistemas dois e três do experimento 1.
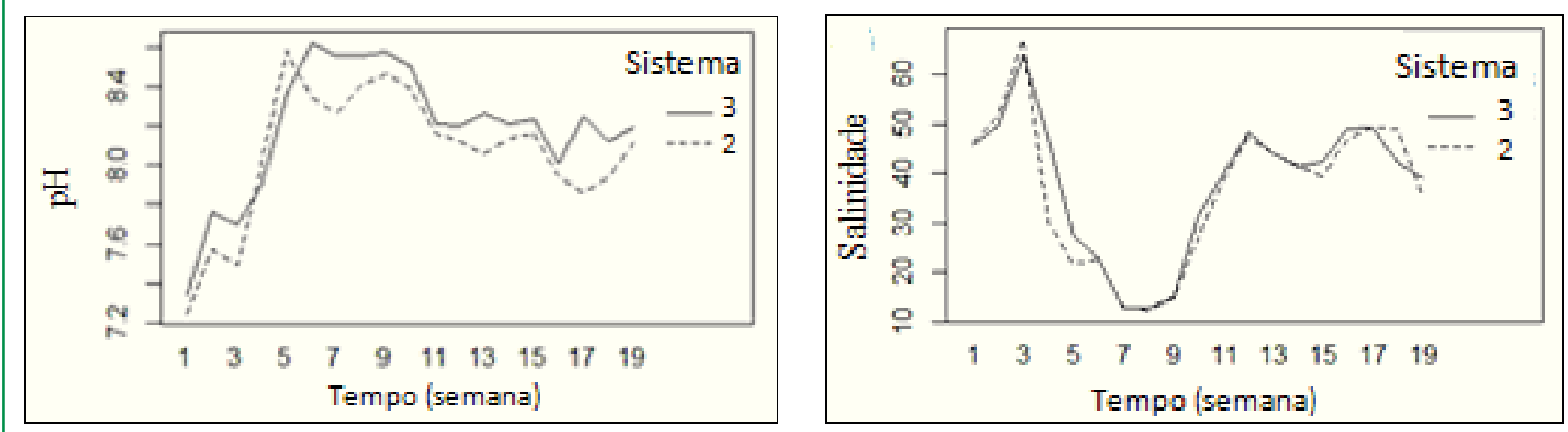

No experimento 2, os parâmetros temperatura, $\mathrm{pH}$, sólidos suspensos totais (SST) e salinidade apresentaram valores médios \pm desvio padrão para o sistema um (sem substrato), dois (areia) e três (areia e plantas) apresentados na Tabela 3.

Temperatura, salinidade e $\mathrm{pH}$ apresentaram diferenças significativas entre os sistemas um, dois e três
(Anexo 1). Amônia, nitrito e ortofosfato em quase todas as medidas mantiveram-se menores no sistema três, com plantas. As concentrações de nitrato e de sólidos suspensos foram maiores no sistema três, seguidos pelo um e dois. Diferenças significativas foram constatadas apenas para concentrações de amônia entre os sistemas um e dois e um e três (Anexo 1).

TABELA 3: Dados médios, desvio padrão e valores mínimo e máximo dos sistemas um, dois e três do experimento 2.

\begin{tabular}{l|c|c|c}
\hline & Sistema um & Sistema dois & Sistema três \\
\hline \multirow{2}{*}{ Temperatura $\left({ }^{\circ} \mathrm{C}\right)$} & $37,9 \pm 0,5$ & $38,1 \pm 0,5$ & $38,6 \pm 0,5$ \\
& $(36,8-41,0)$ & $(36,9-41,0)$ & $(37,1-41,5)$ \\
\hline \multirow{2}{*}{$\mathrm{pH}$} & $8,1 \pm 0,3$ & $8,2 \pm 0,3$ & $8,3 \pm 0,3$ \\
& $(7,7-9,5)$ & $(7,9-9,5)$ & $(7,9-9,5)$ \\
\hline \multirow{2}{*}{ Salinidade (psu) } & $30,6 \pm 0,5$ & $32,1 \pm 0,5$ & $32,1 \pm 0,5$ \\
& $(23,6-38,2)$ & $(23,3-41,6)$ & $(24,2-42,8)$ \\
\hline \multirow{2}{*}{ Sólidos suspensos totais (ppt) } & $23,4 \pm 2,6$ & $24,8 \pm 3,0$ & $24,8 \pm 2,9$ \\
& $(18,9-29,3)$ & $(18,1-31,3)$ & $(19,4-32,1)$ \\
\hline \multirow{2}{*}{ Sólidos suspensos (mg/L) } & $52,7 \pm 26,9$ & $35,9 \pm 20,7$ & $(14,3-89,5)$ \\
\hline \multirow{2}{*}{ Amônia (mg/L) } & $(9,7-82,5)$ & $(4,0-70,0)$ & $0,3 \pm 0,1$ \\
& $0,3 \pm 0,1$ & $0,6 \pm 0,2$ & $(0,2-0,4)$ \\
\hline \multirow{2}{*}{ Nitrito (mg/L) } & $(0,2-0,4)$ & $(0,4-1,0)$ & $0,8 \pm 1,5$ \\
& $1,4 \pm 2,7$ & $0,6 \pm 0,6$ & $(0,0-4,3)$ \\
\hline \multirow{2}{*}{ Nitrato (mg/L) } & $(0,1-7,7)$ & $(0,0-1,6)$ & $1,4 \pm 0,6$ \\
\hline \multirow{2}{*}{ Ortofosfato (mg/L) } & $0,7 \pm 0,4$ & $0,9 \pm 0,6$ & $(0,7-2,5)$ \\
\hline
\end{tabular}


A média quinzenal de evaporação foi de $17,0 \mathrm{~L}$, $13,8 \mathrm{~L}$ e $19,7 \mathrm{~L}$, respectivamente, nos tanques um, dois e três.

\section{Halófitas}

A sobrevivência das mudas de $B$. maritima nos sistemas um e dois do primeiro experimento foi de $73,3 \%$ e $90 \%$, respectivamente. De sessenta mudas da espécie S. virginicus do sistema um restaram sete e do sistema dois, três espécimes.

No experimento 2, as taxas de sobrevivência da B. maritima, $S$. ambigua e $S$. virginicus foram, respectivamente, $91,7 \%, 100 \%, 75 \%$. As mudas de $S$. virginicus que sobreviveram estavam com as folhas secas e o sistema radicular bem desenvolvido.

As Taxas de Crescimento Absoluto (TCA) e de Crescimento Relativo (TCR) (Figura 4) referentes à biomassa da $B$. maritima foram maiores para as plantas que receberam gua residu ria.

FIGURA 4: TCA e TCR, referente à biomassa da B. maritima.

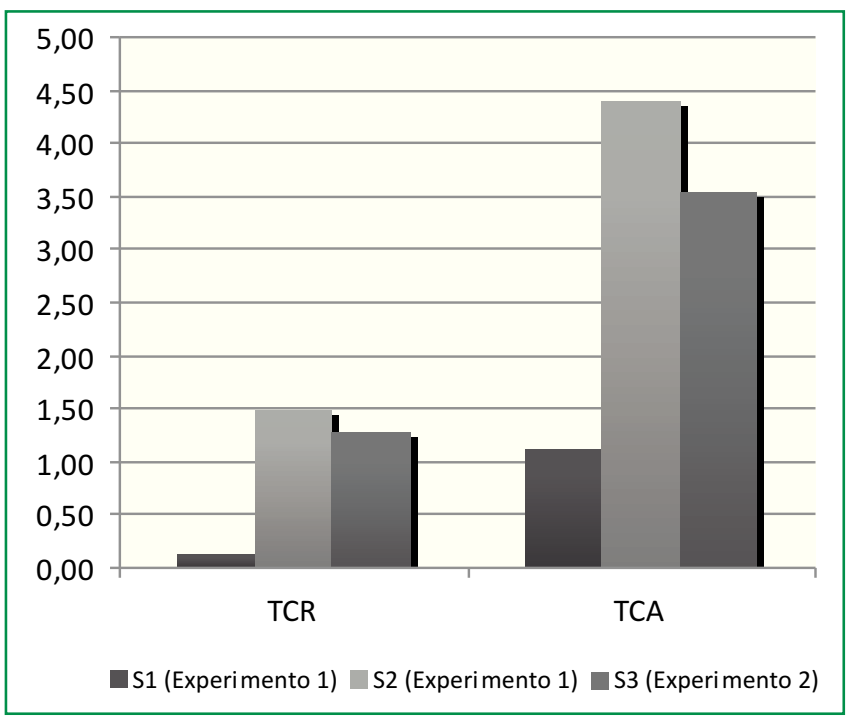

As mesmas taxas calculadas para altura das plantas, em centímetros, apresentaram resultados expressos na Figura 5 (TCR e TCA).
FIGURA 5: Taxas de Crescimento Relativo (TCR) e Absoluto (TCA), referentes à altura de $B$. marítima.

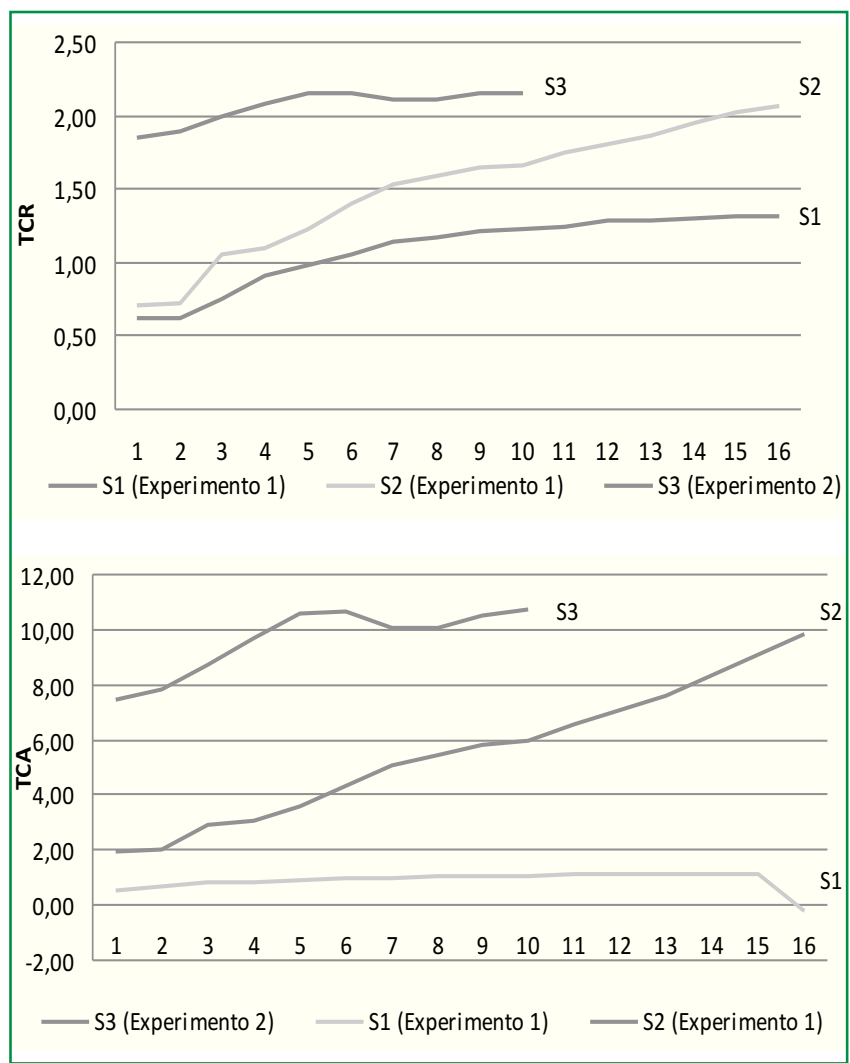

O crescimento médio de altura para as plantas que receberam guas residu rias e para as plantas que receberam água, no experimento 1 , foram significativamente diferentes $(\mathrm{p}=0,01)$.

\section{Litopenaeus vannamei}

A média do peso inicial e final, ganho de biomassa, biomassa final e taxa de sobrevivência dos camarões dos sistemas um, dois e três, do experimento 2, estão expressos na Tabela 4. O volume de água nos tanques de criação de camarão apresentou perda maior no tanque três, ficando com 2,5 L a menos do que no um e 4,9 L a menos do que no dois, de acordo com a média calculada a cada quinze dias. 
TABELA 4: Média do desempenho biométrico e sobrevivência de L. vannamei com densidade de 260 camarões por $\mathrm{m}^{3}$.

\begin{tabular}{l|c|c|c} 
& Sistema 1 & Sistema 2 & Sistema 3 \\
\hline Média do peso inicial $(\mathrm{g})$ & $2,8 \pm 0,9$ & $4,25 \pm 0,3$ & $4,78 \pm 0,6$ \\
\hline Média do peso final $(\mathrm{g})$ & $10,6 \pm 1,5$ & $11,7 \pm 1,4$ & $9,8 \pm 0,8$ \\
\hline Ganho de biomassa $(\mathrm{g})$ & 7,8 & 7,5 & 5,0 \\
\hline Biomassa final $\left(\mathrm{g} / \mathrm{m}^{3}\right) 237,5248,9$ & 237,5 & 248,9 & 231,7 \\
\hline Sobrevivência (\%) & 100,0 & 86,7 & 100,0 \\
\hline
\end{tabular}

\section{Discussão}

\section{Variáveis físico-químicas}

Na China, no Instituto de Pesquisa Pesqueira de Xangai (SFRI), num experimento com sistema de recirculação de água de tanque de criação de camarão em wetland, os pesquisadores verificaram aumento do pH nas áreas úmidas com cultivo de espécies halófitas e valores menores no ponto de coleta de saída $(7,9 \pm 0,5)$ em comparação ao ponto de coleta de entrada $(8,2 \pm 0,6)$ da wetland (SHI et al., 2011). A diminuição constatada no $\mathrm{pH}$ por Shi et al. (2011) foi relacionada ao processo de nitrificação pela disponibilização de íons de $\mathrm{H}^{+}$e, consequentemente, redução do $\mathrm{pH}$ pelo processo de acidificação. Segundo Reeder (1994), a decomposição de material orgânico reduz o oxigênio dissolvido e aumenta o $\mathrm{CO}_{2}$, resultando na redução do $\mathrm{pH}$. Nesse caso, para o experimento 1, mesmo os valores sendo próximos ao reportado por SHI et al. (2011), não foi constatada diferença significativa entre entrada e saída dos sistemas.

Alguns processos propiciados pelas plantas podem compensar a variação de alguns parâmetros, tais como a absorção de $\mathrm{CO}_{2}$ dissolvido através da fotossíntese, aumentando o $\mathrm{pH}$ que, por sua vez, é reduzido pela nitrificação (LIN et al., 2003). Além disso, as bactérias oxidadoras de amônia e bactérias oxidadoras de nitrito podem ter o metabolismo ativado ou não dependendo dos valores do $\mathrm{pH}$, da temperatura e da concentração de oxigênio dissolvido (ZOPPAS et al., 2016).

Shi et al. (2011) e Lin et al. (2005) consideram que o aumento do $\mathrm{pH}$, em reas úmidas, estão relacionados ao processo fotossintético das macrófitas e algas devido à absorção de $\mathrm{CO}_{2}$. Nesse caso, o experimento 2 do sistema com plantas pode estar indicando ação fotossintética das halófitas, pois o sistema com plantas apresentou valores de $\mathrm{pH}$ maiores $(8,3 \pm 0,3)$ do que nos sistemas sem plantas (sistema um 8,1 $\pm 0,3$ e sistema dois $8,2 \pm 0,3$ ), embora a diferença não tenha sido significativa $(p>0,05)$.

No experimento 2, os parâmetros sólidos suspensos, nitrito, nitrato e ortofosfato não apresentaram diferenças significativas entre os tratamentos; assim como no experimento utilizando sistema aquapônico com camarão realizado por Pinheiro et al. (2017), em sistema aquapônico com camarão.

Silva (2016) testou o crescimento da halófita $S$. ambigua exposta a diferentes níveis de estresse hídrico em sistemas de aquaponia com $L$. vannamei. No estudo não foram constatadas diferenças significativas para os parâmetros temperatura, oxigênio dissolvido, alcalinidade, $\mathrm{pH}$, sólidos suspensos totais e ortofosfato. Embora diferenças significativas tenham sido detectadas, pelo autor previamente citado, referentes a salinidade, amônia, nitrito e nitrato em função das semanas de vigência do experimento.

Nos dois experimentos, os maiores teores de salinidade foram observados nos sistemas em que ocorreu maior evaporação de água. De acordo com Sousa et al. (2011), o armazenamento de sal ocorre devido a processos de evaporação. Silva (2016) também relacionou o aumento de salinidade à evaporação da água dos tanques de tratamento.

A evaporação foi maior nos sistemas com cultivo de plantas dos dois experimentos, o que era esperado devido ao processo de evapotranspiração que ocorre 
nos vegetais. Evapotranspiração é a combinação de dois fenômenos: a evaporação, que consiste no processo físico de transferência da gua presente no solo para a atmosfera, e a transpiração, processo fisiológico em plantas no qual a gua sai através dos estômatos das folhas para o meio ambiente (RAJI et al., 2015).

No experimento de Henry-Silva e Camargo (2008), a remoção de nutrientes, como fósforo e nitrogênio, e a turbidez, entre as unidades experimentais, foram menores nas unidades controle, ou seja, sem o cultivo das macrófitas. No caso do experimento 1, o valor médio de ortofosfasto na saída dos sistemas dois e três é bem próximo, ficando explícito que não há diferenças significativas para presença/ausência de plantas na concentração do fósforo.

No experimento 2, com recirculação da água residu ria em tanques com camarão, a amplitude dos valores de ortofosfato foram bem maiores no decorrer das semanas quando comparados ao experimento 1 , devido à constante atividade metabólica dos camarões, não sendo possível comparar os valores mensurados entre os dois experimentos.

A redução percentual do fósforo livre na presença de Salicornia europaea foi de 27\%, (WEBB et al., 2012). No experimento de 2016, desta pesquisa, o percentual de redução de ortofosfato foi maior em dez resultados para o sistema dois e em oito resultados para o sistema três. Os três maiores valores calculados de redução percentual do sistema com plantas, foram de $87,2 \%, 80,0 \%$ e 60,5 e do sistema três foram de $84,6 \%, 84,5$ e $78 \%$.

$\mathrm{O}$ fato de as análises químicas para compostos nitrogenados, ortofosfato e sólidos suspensos do experimento 2 serem realizadas quinzenalmente com cinco semanas de dados levantados dificultaram a constatação mais precisa e consistente das variações desses nutrientes ao longo do tempo. Aliado a isso, os sistemas dois e três apresentaram maior evaporação no volume de gua residu ria, o qual era reposto, influenciando a concentração da matéria orgânica e os parâmetros físicos do fluído.

Fatores que podem interferir nos resultados do tratamento de guas residu rias em wetlands, segundo Henry-Silva e Camargo (2008), são o dimensionamento de tanques experimentais, as espécies cultivadas, as características da gua residu ria utilizada e o sistema de cultivo, se será integrado ou não.

Todos esses fatores estão interligados e há necessidade de experimentos que possam medir a maior quantidade de variáveis possível com número de amostras significativas para possibilitar análises multifatorais, mas isso requer investimento de recurso financeiro, de tempo e de pessoas, o que não foi possível no presente estudo. O que foi possível observar é que nem todos os parâmetros do experimento 2 estavam dentro das faixas recomendadas para o cultivo de camarão, como segue na Tabela 5 (VAN WYK; SCARPA, 1999).

A temperatura ficou acima de $36^{\circ} \mathrm{C}$ em todos os sistemas. $\mathrm{O} \mathrm{pH}$ mensurado apresentou valor máximo de 9,5 e em outras medições, no decorrer do experimento, valores entre 9,0 e 9,3. Segundo Sá (2012), a medida de pH ideal para criação de camarão é de 7,4 por assemelharse ao valor ideal para a atividade enzim tica do animal, mas valores na faixa de 6,5 a 9,0 são considerados ideais para o desenvolvimento do camarão em cultivos. Valores constatados para salinidade estavam acima de 35. A amônia e o nitrito alcançaram valores bem acima do indicado, chegando a níveis considerados letais.

TABELA 5: Parâmetros recomendados de qualidade da água para cultivo de camarão.

\begin{tabular}{l|c}
\multicolumn{1}{c|}{ Parâmetro } & Recomendado \\
\hline Temperatura $\left({ }^{\circ} \mathrm{C}\right)$ & $28,0-32,0$ \\
\hline $\mathrm{pH}$ & $7,0-8,3$ \\
\hline Oxigênio dissolvido $(\mathrm{mg} / \mathrm{L})$ & $5,0-9,0$ \\
\hline Salinidade $(\mathrm{ppt})$ & $0,5-35,0$ \\
\hline Amônia $(\mathrm{mg} / \mathrm{L})$ & 0,03 (máximo) \\
\hline Nitrito $(\mathrm{mg} / \mathrm{L})$ & 1,0 (máximo) \\
\hline Nitrato $(\mathrm{mg} / \mathrm{L})$ & 60,0 (máximo) \\
\hline
\end{tabular}

Fonte: Adaptado de Van Wyk e Scarpa (1999).

\section{Halófitas}

A alta taxa de mortalidade das plantas da espécie $S$. virginicus nos dois experimentos leva à pergunta de 
quais níveis de salinidade são suportados pela planta. O estudo realizado para testar o efeito da salinidade sobre a $S$. virginicus na Universidade do Arizona, USA, demonstrou que as plantas crescidas a 100-150 $\mathrm{mmol} / \mathrm{L}$ de $\mathrm{NaCl}$ eram, visivelmente, maiores do que as plantas cultivadas a $5 \mathrm{mmol} / \mathrm{L}$; além disso, apresentaram ganho de biomassa e aparecimento de perfilhos maiores quando cultivadas a níveis de $\mathrm{NaCl}$ de $450 \mathrm{mmol} / \mathrm{L} \mathrm{em}$ vez de $5 \mathrm{mmol} / \mathrm{L}$ (BELL; O'LEARY, 2003). O estudo confirmou que a $S$. virginicus é uma halófita verdadeira e que apresenta glândulas secretoras de sal em resposta à salinidade. No experimento 1, a média das concentrações de $\mathrm{NaCl}$ na entrada dos sistemas um e dois foram, respectivamente, $440 \mathrm{mmol} / \mathrm{L} \mathrm{e} 450 \mathrm{mmol} / \mathrm{L}$. E na saída, respectivamente, $614 \mathrm{mmol} / \mathrm{L}$ e $650 \mathrm{mmol} / \mathrm{L}$ para os sistemas um e dois. No experimento 2, a média foi de $549 \mathrm{mmol} / \mathrm{L}$, sendo o valor maior do que o do estudo.

O c lculo das medidas de crescimento da altura de $B$. maritima para média, TCA e TCR foi maior nos sistemas com água residuária nos dois experimentos do que no sistema irrigado com gua salgada do rio no experimento 1 . O teste de correlação, para verificar se a água residuária influenciou diretamente na TCA, no experimento 1 , constatou que não foi significativo $(p>0,05)$ nem houve diferença significativa para os valores de salinidade na entrada dos sistemas com plantas.

$\mathrm{O} \mathrm{pH}$ nos dois sistemas com plantas foi entre $6,8 \mathrm{e}$ 8,5 com apenas uma medida abaixo de 7,5, no sistema tratado com gua do rio. $\mathrm{O} \mathrm{pH}$ elevado do mar, entre 7,5 e 8,4, por dificultar a absorção de micronutrientes, pode reforçar o problema de baixa disponibilidade destes (BUHMANN et al., 2015). O pH considerado ótimo para a cultura de plantas hidropônicas está entre 5,5 e 6,5 por evitar a precipitação de nutrientes importantes (BUHMANN et al., 2015). Não foi o caso em ambos os experimentos, nos quais os menores valores de $\mathrm{pH}$, em que havia cultivo de plantas, foi maior que 7,2. Embora as plantas dos experimentos não tenham sido cultivadas em hidroponia, em alguns momentos, as raízes ficaram submersas.

A TCR, do crescimento em altura das plantas $B$. maritima, e as medidas de $\mathrm{pH}$, do experimento 1 , foram plotadas num gráfico de dispersão para estimativa da correlação; posteriormente, foi testada a significância da relação pelo método de Pearson para amostras paramétricas. $\mathrm{O}$ resultado não apresentou correlação significativa alta $(p=0,21)$. No experimento 2 não foi possível aplicar os testes de correlação, pois os dados não apresentaram normalidade nem homogeneidade.

No teste de hipótese do experimento 1, aplicado para detectar se houve diferença significativa da taxa absoluta de crescimento entre as plantas tratadas com gua do rio e as plantas tratadas com gua residu ria, foi constatada diferença significativa $(p=0,01)$, sendo o crescimento maior para as plantas tratadas com gua residuária. $\mathrm{O}$ fator que pode ter influenciado é o aporte maior de nutrientes na gua residu ria do que na gua do rio. As guas residu rias de carcinicultura aumentam significativamente a clorofila a, o nitrogênio inorgânico, o total de nitrogênio e o fósforo na coluna de água (MOLNAR et al., 2013).

No experimento 1, o ortofosfato nos sistemas um e dois apresentaram diferença significativa $(p=0,001)$ e os maiores valores constatados na gua residu ria. No sistema em que as plantas foram irrigadas com água do Rio Pacoti foi possível constatar que quase todas as concentrações de ortofosfato estavam abaixo de 0,3 mg/L. O experimento de Buhmann et al. (2015) demonstrou que a quantidade de ortofosfato entre 0,3 e 1,6 mg/L não prejudica a produção de biomassa e a absorção de nitrogênio, mas as concentrações entre 1,6 e 3,3 mg/L são mais favoráveis para a absorção de ortofosfato.

A espécie $S$. ambigua apresentou ganho de biomassa reduzido, pois o crescimento limitou-se ao alongamento apenas da haste principal na maioria das plantas.

Os dados levantados do experimento 1 demonstraram que as plantas da espécie $B$. maritima irrigadas com gua residu ria de carcinicultura cresceram mais dos que as cultivadas com gua do rio Pacoti. Estudos com dimensionamento maior dos sistemas são necessários para verificar se há influência na taxa de sobrevivência da espécie $S$. virginicus.

As águas residuárias do experimento 2, que recircularam pelas plantas, apresentaram menor 
quantidade de acúmulo de nutrientes do que na ausência de plantas. Faz-se necess rio estudo com mais amostragens para poder compreender melhor as interações dos camarões, das plantas e da água residuária.

\section{Litopenaeus vannamei}

A média final do peso dos camarões e a taxa de sobrevivência estavam próximas das médias encontradas em outros estudos (PINHEIRO, 2015; SILVA, 2016), embora o ganho de biomassa estivesse abaixo. O peso inicial foi menor no tanque 1 , porém o ganho de biomassa deste foi similar ao do tanque 2. Ressalta-se que o tanque 2 apresentou significativamente mais amônia que o tanque 1, além de outros parâmetros não tão destacados. Os camarões do tanque 3 apresentaram menor ganho de biomassa em relação aos outros, apresentando valores significativamente maiores que o tanque 1 em relação a temperatura, $\mathrm{pH}$, salinidade $\mathrm{e}$ sólidos suspensos totais, embora essas diferenças não tenham sido tão marcantes.

Então, para este estudo, o ganho de biomassa foi mais favorecido pelo sistema de recirculação contínua da gua residu ria sem a presença de substrato do que somente com substrato e substrato com plantas, embora não sido testado o uso de plantas sem substrato e seu efeito no ganho da biomassa do camarão. Possivelmente, a presença dos substratos brita e areia não seja o ideal para aquaponia sem o uso de um biofiltro para estimular a nitrificação. Isso reporta a necessidade de outros estudos que venham a testar o efeito de substratos em aquaponia.

Embora a maioria dos parâmetros físico-químicos estivesse fora dos padrões ideais para a produção, a taxa de sobrevivência dos camarões manteve-se alta, acima de $85 \%$.

De forma geral, o experimento 1 possibilitou visualizar que o cultivo de plantas é beneficiado pela irrigação com gua residu ria da carcinicultura, porém sua presença/ausência não alterou os parâmetros avaliados (i.e., temperatura, $\mathrm{pH}$, salinidade, oxigênio dissolvido, sólidos suspensos totais, sólidos suspensos e ortofosfato). Já no segundo experimento, com uso da gua da carcinicultura em sistema de recirculação, o desempenho zootécnico do camarão foi melhor sem a presença de substrato e com o substrato com plantas, destacando-se que as condições desse experimento não foram ideais para a produção, devido aos altos valores de amônia e nitrito, bem como não foi testado o uso de plantas sem substrato.

Contrastando os dois experimentos, pode-se observar que as plantas foram maiores quando irrigadas com água residuária no primeiro experimento do que com água do efluente em sistema de recirculação. Não é possível sugerir que a produção vegetal é maior em sistemas desacoplados do que em sistemas acoplados, pois o tempo de cultivo foi maior no experimento 1 , com dez dias de diferença.

\section{Agradecimentos}

À ACP Engenharia, ao Depósito Alvorada e ao Depósito Claudio, pela doação de materiais que serviram para a montagem dos experimentos, e ao Professor Alberto Nunes, por ceder o espaço no CEAC para a realização dos experimentos.

\section{Referências}

BELL, H. L.; O'LEARY, J. W. Effects of salinity on growth and cation accumulation of Sporobolus virginicus (Poaceae). American Journal of Botany, St. Louis, v. 90, n. 10, p. 1416-1424, 2003.

BERTIN, R. L.; GONZAGA, L. V.; BORGES, G. S. C.; AZEVEDO, M. S.; MALTEZ, H. F.; HELLER, M.; MICKE, G. A.; TAVARES, L. B. B.; FETT, R. Nutrient composition and, identification/quantification of major phenolic compounds in Sarcocornia ambigua (Amaranthaceae) using HPLC-ESI-MS/MS. Food Research International, Campinas, v. 55, p. 404-411, 2014. BIUDES, J. F. V. Tratamento de efluentes de carcinicultura. 2007. 121 f. Tese (Doutorado em Aquicultura) - Universidade Paulista, Jaboticabal. 2007.

BRASKERUD, B. C. Factors affecting nitrogen retention in small constructed wetlands treating agricultural non-point source pollution. Ecological Engineering, Columbus, v. 18, p. 351-370, 2002.

BUHMANN, A.; PAPENBROCK, J. Biofiltering of aquaculture effluents by halophytic plants: Basic principles, current uses and future perspectives. Environmental and Experimental Botany, Paris, v. 92, p. 122-133, 2013.

BUHMANN, A. K.; WALLER, U.; WECKER, B.; PAPENBROCK, J. Optimization of culturing conditions and selection of species for the use of halophytes as biofilter for nutrient-rich saline water. Agricultural Water Management, Amsterdam, v. 149, p. 102114,2015 
D'OCA, M. G. M.; MORÓN-VILLARREYES, J. A.; LEMÕES, J. S.; COSTA, C. S. B. Fatty acids composition in seeds of the South American glasswort Sarcocornia ambigua. Anais da Academia Brasileira de Ciências, Rio de Janeiro, v. 84, n. 3, p. 865-870, 2012.

FABIANO, R. B. Conflitos socioambientais e gestão integrada e sustentável de recursos pesqueiros. 2004. 102 f. Dissertação (Mestrado em Sociologia Política) - Universidade Federal de Santa Catarina, Florianópolis. 2004.

FLOWERS, T. J.; COLMER, T. D. Salinity tolerance of halophytes. New Phytologist, Lancaster, v. 179, p. 945-963, 2008.

HASANUZZAMAN, M.; NAHAR, K.; ALAM, M. M.; BHOWMIK, P. C.; HOSSAIN, M.A.; RAHMAN, M. M.; PRASAD, M. N. V.; OZTURK, M.; FUJITA, M. Potential use of halophytes to remediate saline soils. Biomed Research International, Cairo, v. 2014, p. 12, 2014.

HENRY-SILVA, G. G.; CAMARGO, F. M. Tratamento de efluentes de carcinicultura por macrófitas aquáticas flutuantes. Revista Brasileira de Zootecnia, Viçosa, v. 37, n. 2, p. 181-188, 2008.

KIVAISI, A. K. The potential for constructed wetlands for wastewater treatment and reuse in developing countries: a review. Ecological Engineering, Columbus, v. 16, p. 545-560, 2001.

KSOURI, R.; KSOURI, W. M.; JALLALI, I.; DEBEZ, A.; MAGNE, C.; HIROKO, I.; ABDELLY, C. Medicinal halophytes: potent source of health promoting biomolecules with medical, nutraceutical and food applications. Critical Review in Biotechnology, Edinburgh, v. 32, p. 289-326, 2012.

LIETH, H.; SUCRE, M. G.; HERZOG, B. Mangroves and halophytes: restoration and utilisation. 1 ed. Amsterdam: Springer Netherlands, 2008. 160 p.

LIN, Y-F.; JING, S-R.; LEE, D-Y. The potential use of constructed wetlands in a recirculating aquaculture system for shrimp culture. Environmental Pollution, Amherst, v. 123, p. 107-113, 2003.

LIN, Y-F.; JING, S-R.; LEE, D-Y.; CHANG, Y. M.; SHIH, K. C. Performance of a constructed wetland treating intensive shrimp aquaculture wastewater under high hydraulic loading rate. Environmental Pollution, Amherst, v. 134, p. 411-421, 2005.

LONARD, I. R.; JUDD, W. F; STALTER, R. The Biological flora of coastal dunes and wetlands: Batis maritima $\mathrm{C}$. Linnaeus. Journal of Coastal Research, Fort Lauderdale, v. 27, n. 6, p. 441449, 2011.

LONARD, I. R.; JUDD, W. F; STALTER, R. The biological flora of coastal dunes and wetlands: Distichlis spicata (C. Linnaeus) E. Greene. Journal of Coastal Research, Fort Lauderdale, v. 29, n. 1, p. 105-117, 2013.

MARCONE, M. F. Batis maritima (Saltwort/Beachwort): a nutritious, halophytic, seed bearings, perennial shrub for cultivation and recovery of otherwise unproductive agricultural land affected by salinity. Food Research International, Campinas, v. 36, p. 123 130, 2003.

MOLNAR, N.; WELSH, D. T.; MARCHAND, C.; DEBORDE, J.; MEZIANE, T. Impacts of shrimp farm effluent on water quality, benthic metabolism and N-dynamics in a mangrove forest (New Caledonia). Estuarine, Coastal and Shelf Science, New York, v. 117, p. 12-21, 2013.

PINHEIRO, I. C. Produção de halófita Sarcocornia ambigua e Litopenaeus vannamei em sistema de aquaponia com bioflocos.
2015. 48 f. Dissertação (Mestrado em Aquicultura) - Universidade Federal de Santa Catarina, Florianópolis. 2015.

PINHEIRO, I. C.; ARANTES, R.; DO ESPÍRITO SANTO, C. M.; DO NASCIMENTO VIEIRA, F.; LAPA, K. R.; GONZAGA, L. V.; FETT, R.; BARCELOS-OLIVEIRA, J. L.; SEIFFERT, W. Q. Production of the halophyte Sarcocornia ambigua and Pacific white shrimp in an aquaponic system with biofloc technology. Ecological Engineering, Columbus, v. 100, p. 261-267, 2017.

QADIR, M.; OSTER, J. D.; SCHUBERT, S.; NOBLE, A. D.; SAHRAWAT, K. L. Phytoremediation of sodic and saline-sodic soils. Advances in Agronomy, San Diego, v. 96, p. 197-247, 2007.

RAJI, B.; TENPIERIK, M. J.; DOBBELSTEEN, A. The impact of greening systems on building energy performance: a literature review. Renewable \& Sustainable Energy Reviews, Pulau Pinang, v. 45, p. 610-623, 2015.

REEDER, B. Assessing constructed wetland functional success using diel changes in dissolved oxygen, $\mathrm{pH}$, and temperature in submerged, emergent, and open-water habitats in the Beaver Creek Wetlands Complex, Kentucky (USA). Ecological Engineering, Columbus, v. 3, p. 161-169, 1994.

RIBEIRO, L. F.; SOUZA, M. M.; BARROS, F. Desafios da carcinicultura: aspectos legais, impactos ambientais e alternativas mitigadoras. Revista da Gestão Costeira Integrada, Itajaí, v. 14, p. 365-383, 2014.

SÁ, M. V. C. Limnocultura: Limnologia para Aquicultura. Fortaleza: Edições UFC, 2012. 218 p.

SHAER, H. M. El. Halophytes and salt-tolerant plants as potential forage for ruminants in the Near East region. Small Ruminant Research, Newark, v. 91, p. 3-12, 2010.

SHI, Y.; ZHANG, G.; LIU, J.; ZHU, Y.; XU, J. Performance of a constructed wetland in treating brackish wastewater from commercial recirculating and super-intensive shrimp growout systems. Bioresource Technology, Oxford, v. 102, p. 9416-9424, 2011.

SILVA, H. V. Efeitos do estresse hídrico na produção de compostos bioativos de Sarcocornia ambigua em sismema aquapônico com Litopenaeus vannamei. 2016. 59 f. Dissertação (Mestado em Aquicultura) - Universidade Federal de Santa Catarina, Florianópolis. 2016.

SOTIROUDIS, V.; SOTIROUDIS, T.; KOLISIS, F. The potential of biodiesel production from fatty acid methyl esters of some European/Mediterranean and cosmopolitan Halophyte seed oils. Journal of ASTM International, Pennsylvania, v. 7, n. 3, p. 1-9, 2010.

SOUSA, W. T. Z.; PANITZ, C. M. N.; THOMAZ, S. M. Performance of pilot-scale vertical flow constructed wetlands with and without the emergent macrophyte Spartina alterniflora treating mariculture effluent. Brazilian Archives of Biology and Technology, Curitiba, v. 54, p. 405-413, 2011.

VAN OOSTEN, M. J.; MAGGIO, A. Functional biology of halophytes in the phytoremediation of heavy metal contaminated soils. Environmental and Experimental Botany, Paris, v. 111, p. 135-146, 2015.

VAN WYK, P.; SCARPA, J. Water quality requirements and management. In: VAN WYK, P.; DAVIS-HODGKINS, M.; LARAMORE, R.; MAIN, K. L.; SCARPA, J. (Ed.). Farming marine shrimp in recirculating freshwater systems. Tallahassee: 
Florida Department of Agriculture and Consumer Services, 1999. P. 141-162.

WEBB, J. M.; QUINTÃ, R.; PAPADIMITRIOU, S.; NORMAN, L.; RIGBY, M. Halophyte filter beds for treatment of saline wastewater from aquaculture. Water Research, London, v. 46, p. $5102-5114,2012$.
ZOPPAS, F. M.; BERNARDES, A. M.; MENEGUZZI, A. Parâmetros operacionais na remoção biológica de nitrogênio de águas por nitrificação e desnitrificação simultânea. Engenharia Sanitária e Ambiental, Rio de Janeiro, v. 21, p. 29-42, 2016.

\section{ANEXO 1: Teste de hipótese para amostras independentes do experimento 2.}

\begin{tabular}{l|c|c|c}
\hline & $\begin{array}{c}\text { Sistema um } \\
\mathbf{x} \\
\text { Sistema dois }\end{array}$ & $\begin{array}{c}\text { Sistema um } \\
\mathbf{x} \\
\text { Sistema três }\end{array}$ & $\begin{array}{c}\text { Sistema dois } \\
\mathbf{x} \\
\text { Sistema três }\end{array}$ \\
\hline Temperatura & $0,00113(* *)$ & $2,843 \mathrm{e}-07(* *)$ & $0,02005(* *)$ \\
\hline $\mathrm{pH}$ & $5,2 \mathrm{e}-5(* *)$ & $3,28 \mathrm{e}-10(* *)$ & $0,00483(*)$ \\
\hline Salinidade & $0,005339(* *)$ & $0,004654(* *)$ & $0,9442\left(^{*}\right)$ \\
\hline Sólidos suspensos totais & $0,004901(* *)$ & $0,004193(* *)$ & $0,9398\left(^{*}\right)$ \\
\hline Sólidos suspensos & $0,1271\left(^{*}\right)$ & $0,7886(*)$ & $0,07805(*)$ \\
\hline Amônia & $0,0002762\left(^{* *}\right)$ & $0,1193(*)$ & $0,0003231\left(^{* *}\right)$ \\
\hline Nitrito & $0,375(*)$ & $0,1986(*)$ & $0,7054(*)$ \\
\hline Nitrato & $0,6272\left(^{*}\right)$ & $0,01363\left(^{*}\right)$ & $0,1027\left(^{*}\right)$ \\
\hline Ortofosfato & $0,5229\left(^{*}\right)$ & $0,4027(*)$ & $0,2609\left(^{*}\right)$ \\
\hline
\end{tabular}

(*): Não apresenta diferença significativa; $(* *)$ Apresenta diferença significativa $(\mathrm{p}<0,05)$. 